\title{
Prevalência das doenças metabólicas em recém-nascidos de Unidade de Terapia Intensiva neonatal submetidos ao teste do pezinho
}

Prevalence of metabolic diseases in newborns of the neonatal Intensive Care Unit submitted to the heel prick test

Prevalencia de enfermedades metabólicas en recién nacidos de la Unidad de Cuidados Intensivos neonatales sometidos a la prueba de punción del talón

Teresa Virgínia Neves Floriano ORCID: https://orcid.org/0000-0003-3713-1664 Universidade Tiradentes, Brasil

E-mail: teresa.virginia@ souunit.com.br

Renata Hellen Silva Andrade ORCID: https://orcid.org/0000-0001-8414-923X Universidade Tiradentes, Brasil

E-mail: renata5hellen@gmail.com

Izailza Matos Dantas Lopes ORCID: https://orcid.org/0000-0001-9752-5628 Universidade Tiradentes, Brasil E-mail: izailzamatos@gmail.com

Caroline Cordeiro Vieira ORCID: https://orcid.org/0000-0001-5537-6855 Universidade Tiradentes, Brasil

E-mail: carolacordeirov@gmail.com Leonardo Santos Melo ORCID: https://orcid.org/0000-0003-0486-6031 Universidade Tiradentes, Brasil E-mail: lekosm11@gmail.com

Laís Costa Matias

ORCID: https://orcid.org/0000-0002-9237-5023 Universidade Tiradentes, Brasil

E-mail: laiscosta201@hotmail.com

Lorenna Oliveira Menezes

ORCID: https://orcid.org/0000-0002-0351-5398 Universidade Tiradentes, Brasil

E-mail: loremenezes@hotmail.com

Mariana Guimarães Nolasco Farias

ORCID: https://orcid.org/0000-0002-3375-2407 Universidade Tiradentes, Brasil E-mail: marianagnf@outlook.com

\begin{abstract}
Resumo
Objetivos: Descrever a prevalência das doenças metabólicas em recém-nascidos de unidade de terapia intensiva neonatal submetidos ao teste do pezinho. Método: Trata-se de um estudo transversal, retrospectivo e analítico, por meio da análise de 261 prontuários de recém-nascidos de maternidade de referência em Sergipe com recém-nascidos que necessitaram de internação em unidade de terapia intensiva neonatal e que faziam seguimento no ambulatório de especialidades da maternidade. Resultados: Dos 261 prontuários avaliados, 105 apresentaram resultado do teste do pezinho. Dentre as doenças triadas nota-se que a dosagem de Fenilalanina se situou dentro da normalidade em todos os pacientes; a positividade para o Hipotireoidismo Congênito foi de 3,8\%, para Fibrose Cística foi de 2\%, para Hiperplasia Adrenal Congênita foi de 1\%, para Deficiência de Biotinidase foi de 4,2\% e para as Hemoglobinopatias foi de 9,5\%. Conclusão: A doença metabólica de maior prevalência nesse estudo foi o grupo que compreende as Hemoglobinopatias triadas pelo teste do pezinho, que correspondem a 9,5\% de resultados positivos. Tal achado corrobora com o perfil de prevalência de doença metabólica no Brasil, com ênfase para a Anemia Falciforme, na triagem neonatal.
\end{abstract}

Palavras-chave: Triagem neonatal; Prevalência; 


\begin{abstract}
Objectives: To describe the prevalence of metabolic diseases in newborns in the neonatal intensive care unit undergoing heel prick testing. Method: This is a cross-sectional, retrospective and analytical study, through the analysis of 261 medical records of newborns of reference maternity in Sergipe with newborns who required hospitalization in a neonatal intensive care unit and who were followed up in the hospital. outpatient clinic of maternity specialties. Results: Of the 261 medical records evaluated, 105 presented results of the heel prick test. Among the diseases screened, it is noted that the dosage of Phenylalanine was within the normal range in all patients; positivity for Congenital Hypothyroidism was 3.8\%, for Cystic Fibrosis it was 2\%, for Congenital Adrenal Hyperplasia it was $1 \%$, for Biotinidase Deficiency it was $4.2 \%$ and for Hemoglobinopathies it was $9.5 \%$. Conclusion: The most prevalent metabolic disease in this study was the group that comprises Hemoglobinopathies screened by the heel prick test, which correspond to $9.5 \%$ of positive results. This finding corroborates the profile of the prevalence of metabolic disease in Brazil, with emphasis on sickle cell anemia, in neonatal screening.
\end{abstract}

Keywords: Neonatal screening; Prevalence; Health profile.

\title{
Resumen
}

Objetivos: Describir la prevalencia de enfermedades metabólicas en recién nacidos en la unidad de cuidados intensivos neonatales sometidos a prueba de punción del talón. Método: Se trata de un estudio transversal, retrospectivo y analítico, mediante el análisis de 261 historias clínicas de recién nacidos de maternidad de referencia en Sergipe con recién nacidos que requirieron hospitalización en una unidad de cuidados intensivos neonatales y que fueron seguidos en el ambulatorio. de especialidades de maternidad. Resultados: De las 261 historias clínicas evaluadas, 105 presentaron resultados de la prueba de punción del talón. Entre las enfermedades examinadas, se observa que la dosis de fenilalanina estuvo dentro del rango normal en todos los pacientes; la positividad para Hipotiroidismo Congénito fue de 3.8\%, para Fibrosis Quística fue de 2\%, para Hiperplasia Suprarrenal Congénita fue de 1\%, para Deficiencia de Biotinidasa fue de 4.2\% y para Hemoglobinopatías fue de 9.5\%. Conclusión: La enfermedad metabólica más prevalente en este estudio fue el grupo que comprende las Hemoglobinopatías cribadas mediante la prueba de punción del talón, que corresponden al 9,5\% de los resultados positivos. Este hallazgo corrobora el perfil de prevalencia de enfermedad metabólica en Brasil, con énfasis en la anemia falciforme, en el cribado neonatal.

Palabras clave: Cribado neonatal; Prevalencia; Perfil de salud.

\section{Introdução}

A assistência pública em saúde no Brasil ofertada através do Sistema Único de Saúde (SUS), como proposto em seus princípios doutrinários e organizacionais, baseia-se na promoção da saúde por um conceito amplo, que envolve principalmente a medicina preventiva. Objetivando a redução do risco de se adquirir uma doença e/ou suas complicações, a prevenção é dividida em níveis prevenção primário, secundário, terciário ou quaternário (Tesser, 2017).

Em nível secundário, diversas ações são realizadas para detectar um problema de saúde em estágio inicial, muitas vezes subclínico, ou qualquer desvio da normalidade, facilitando o diagnóstico e tratamento ou prevenindo sua disseminação e efeitos a longo prazo. Dois grandes exemplos de prevenção secundária são o rastreamento de doenças e o diagnóstico precoce (Brasil, 2013).

É importante, entretanto, diferenciar rastreamento e diagnóstico de doenças. Quando o indivíduo exibe sinais e sintomas de uma doença e um teste diagnóstico é realizado, este não representa um rastreamento. No diagnóstico de doenças, realiza-se os exames necessários de acordo com a clínica apresentada pelo paciente, o que não configura rastreamento, mas sim cuidado e diagnóstico apropriado (Engelgau, el al., 2000). Já no rastreamento, exames ou testes são aplicados em pessoas presumidamente sadias, o que implica, repetimos e enfatizamos, garantia de benefícios relevantes frente aos riscos e danos previsíveis (Brasil, 2013). Assim, o rastreamento servirá como instrumento para triar e classificar os indivíduos suspeitos ou não de determinada doença. No caso de suspeito, deve-se obrigatoriamente seguir com outros exames para que se chegue ao diagnóstico final (Brasil, 2011).

Nesse contexto, o teste de triagem neonatal (TN), popularmente conhecido como teste do pezinho (TP), foi adotado como medida que objetiva rastrear doenças metabólicas em RN e evitar danos irreversíveis durante o seu desenvolvimento. 
Seu surgimento data de final da década de 50, tendo os Estados Unidos como pioneiros, em pesquisas que visavam prevenir doenças mentais em RN (Pilar, 2018). Porém, foi somente em 1963 que o médico microbiologista Robert Guthrie fomentou o desenvolvimento do teste, com a finalidade de rastreamento metabólico, tendo em vista a prevenção da fenilcetonúria (Zhu, 2017). A primeira técnica, popularmente conhecida como "teste de Guthrie" era feita por inibição bacteriana em amostras de sangue e colhidas em papel-filtro para avaliação dos níveis de fenilalanina.

No Brasil, a evolução da testagem foi relativamente semelhante. Os primeiros indícios foram em São Paulo no ano de 1976, com o rastreamento de PKU, evoluindo em 1980 com detecção também de hipotireoidismo congênito (Leão, 2008). Entretanto, apenas em 2001 houve a criação do Programa Nacional de Triagem Neonatal (PNTN), tornando-se a maior iniciativa do SUS na área genética, ampliando a triagem já existente, produzindo dados que possibilitam uma abordagem efetiva da situação brasileira (Botler, et al., 2010).

Nos estados brasileiros, a TN é implantada em quatro fases: Fase I - Triagem em Fenilcetonúria (PKU) e Hipotireoidismo Congênito (HC), Fase II - Exames da fase I e triagem para Anemia Falciforme (AF) e outras hemoglobinopatias (HP); Fase III - Exames da fase II e triagem para Fibrose Cística (FC), Fase IV - Exames da fase III e triagem para Hiperplasia Adrenal Congênita (HAC) e para Deficiência da Biotinidase (DB) (Corrêa, 2019). Além dessas doenças cobertas pela rede pública, existe o teste do pezinho ampliado, oferecido pela rede privada, que inclui hemocistinúria, hiperfenilalaninemia e deficiência de TBG (globulina carregadora de tirosina) (Cruz, 2014). Como o teste ampliado não está disponível na rede pública, dificulta o acesso de pessoas mais carentes à detecção de doenças mais raras (Camargo, et al., 2019). Segundo o Ministério da Saúde, o teste deve ser realizado preferencialmente entre o $3^{\circ}$ e $5^{\circ}$ dia de vida, não sendo recomendado ultrapassar 30 dias.

A PKU resulta da deficiência da fenilalanina hidroxilase, enzima que catalisa a conversão de fenilalanina em tirosina. Existem diversas variantes da PKU, caracterizadas entre a completa ausência de atividade e 5\% de atividade residual, associada respectivamente a altas concentrações de fenilalanina no plasma $\left({ }^{3} 1.200 \mathrm{mmol} / \mathrm{L}\right)$ e concentrações normais (Guttler, 1996). As hiperfenilalaninemias (HPA) podem ser classificadas em PKU clássica, PKU leve ou HPA não PKU, a depender do nível de fenilalanina sérica ao diagnóstico. Tal nível fornece uma estimativa da atividade enzimática residual que, em parte, depende da mutação presente no gene permite a definição do fenótipo bioquímico (de Marqui, 2017). O tratamento da PKU baseia-se na restrição dietética de fenilalanina e no uso de fórmula metabólica rica em aminoácidos necessários para o crescimento. Tal tratamento deve ser instituído o mais precocemente possível, preferencialmente até o décimo dia após o nascimento. Dessa forma, os níveis séricos de fenilalanina tendem a diminuir, evitando o dano neurológico (Brasil, 2019).

Outra doença diagnosticada pelo TP, a fibrose cística é uma doença genética autossômica recessiva caracterizada pela disfunção do gene cystic fibrosis transmembrane conductance regulator (CFTR), que codifica uma proteína reguladora de condutância transmembrana de cloro (Athanazio, et al., 2017).

Já a deficiência de biotinidase é um erro inato do metabolismo, de origem genética e herança autossômica recessiva que consiste na deficiência da enzima biotinidase, responsável pela absorção e regeneração orgânica da biotina, vitamina existente em alimentos comuns, como fígado, leite, ovos e carnes (Arantes, et al., 2016). Segundo dados do Ministério da Saúde, cerca de $20 \%$ dos casos estão associados a consanguinidade, possuindo uma incidência geral de 1/60.000 nascidos vivos. A biotina - vitamina $\mathrm{B} 7$ ou vitamina $\mathrm{H}$ - é vitamina do complexo $\mathrm{B}$, hidrossolúvel, fundamental para a efetivação de processos metabólicos orgânicos como gliconeogênese, síntese de ácidos graxos e catabolismo de aminoácidos de cadeia ramificada. Atua como coenzima das carboxilases humanas: piruvato-carboxilase, propionil-CoA carboxilase, B-metilcrotonilCo-A carboxilase e acetil-CoA1 (Wollf, 2001). Os sintomas são variáveis, assim como o início das manifestações, incluindo alopecia, atraso no desenvolvimento, hipotonia muscular, crises epilépticas, rash cutâneo com descamação, infecções de pele, ataxia, conjuntivite, deficiência auditiva, letargia, problemas respiratórios, distúrbios visuais, dificuldades de alimentação, 
diarreia e infecções. Nesse contexto, o tratamento é feito com a reposição oral de biotina de 5 a 30mg/dia (Arantes, et al., 2016).

A maioria dos casos de $\mathrm{HC}$ é decorrente de disgenesias tireoidianas, principalmente a ectopia, hipoplasia ou agenesia tireoidianas, e os demais casos são resultados de defeitos de síntese hormonal (Maciel, et al., 2013). O teste de triagem neonatal serve como importante meio de suspeição do HC, sendo necessário para o seu diagnóstico a confirmação por métodos quantitativos de rotina para as dosagens de concentrações séricas de TSH e T4 total ou T4 livre (Zilka, 2008). Nesse contexto, o diagnóstico precoce oferece a possibilidade de idade de iniciar o tratamento na idade adequada, com monitoramento da dose de levotiroxina, essenciais para um bom prognóstico (Lafranchi, 2011).

A hiperplasia adrenal congênita é o termo utilizado para conceituar um conjunto de doenças, de herança autossômica recessiva, resultantes da deficiência da enzima 21-hidroxilase, responsável pela síntese de cortisol nas glândulas adrenais. A triagem possibilita o diagnóstico precoce da doença, prevenindo a gravidade da crise perdedora de sal, além de evitar complicações tardias, principalmente as associadas à hiponatremia neonatal, tais como retardo mental e distúrbios cognitivos (Kopacek, et al., 2017). O acompanhamento dos bebês afetados deve ser multidisciplinar. Depois de iniciado a terapêutica, recomenda-se o acompanhamento semanal durante os primeiros 2 meses de vida, com dosagens de sódio e potássio (SPB, 2019).

Por fim, as hemoglobinopatias são as alterações mais comuns observadas no teste de triagem neonatal. São um grupo heterogêneo de doenças hereditárias, na maioria autossômica recessivas, resultando em mutações representadas pelas hemoglobinas S e C (HbS e HbC) e pelas alfa e beta talassemias (Kohne, 2011). Estima-se a existência de dois milhões de indivíduos com traço falciforme no Sudeste e Nordeste, que são as acometidas (Silva, et al., 2015).

Apesar de a incidência isolada de cada uma dessas doenças ser pequena, em virtude de sua origem autossômica recessiva, quando analisadas em conjunto, elas equivalem a cerca de $10 \%$ das doenças genéticas e vêm aumentando proporcionalmente à diminuição da letalidade por doenças infecciosas e distúrbios nutricionais (Levy, 1998). Somado a isso, não existem dados públicos oficiais do Ministério da Saúde (MS), que mostre a frequência dessas doenças na população brasileira, dificultando a compreensão da evolução dos casos e inviabilizando o fortalecimento de políticas públicas direcionadas.

Diante da pesquisa realizada, este presente trabalho objetiva a compreensão da Prevalência das doenças metabólicas em recém-nascidos de UTIN submetidos ao teste do pezinho, na tentativa de melhor conhecer o cenário epidemiológico e possibilitar melhores intervenções.

\section{Metodologia}

Trata-se de um estudo observacional, transversal, retrospectivo, descritivo e quantitativo (Pereira et al., 2018) por meio da análise de prontuários de RN de uma maternidade de ensino no município de Aracaju (SE). O estudo ocorreu em uma maternidade de referência em Sergipe com RN que necessitaram de internação em UTIN e que faziam seguimento no ambulatório de especialidades da maternidade. Utilizou-se um instrumento para a pesquisa elaborado pelos pesquisadores que possuía variáveis dos RN acerca da realização ou não do TP com os respectivos valores detectados. Foram incluídos prontuários físicos de crianças nascidas no período delimitado de janeiro de 2018 a junho de 2020 que possuíam as variáveis analisadas.

Os dados foram compilados no programa Microsoft Office Excel. Foram obtidas variáveis quantitativas. Para as variáveis qualitativas a análise descritiva procedeu com a categorização dos dados e obtenção das respectivas frequências e percentuais e o cálculo da média, mediana, desvio padrão, mínimo e máximo das variáveis quantitativas. O tamanho da 
amostra foi calculado com base da média de atendimento mensal do ambulatório de especialidades da maternidade do estudo considerando um erro de $5 \%$ e um nível de confiança de $95 \%$. Para a realização deste cálculo foi considerado uma população finita (N) de 200 pacientes, desvio padrão de 0,3 (alfa), Z (variável normal padronizada associada ao nível de confiança =1,96) e o erro amostral de 0,05 (E). Resultando em um total de 136 prontuários, que foi incrementado para 261 participantes para compensar qualquer perda potencial e aumentar o poder do estudo.

O projeto foi submetido à apreciação e avaliação do Comitê de Ética em Pesquisa (CEP) da Universidade Tiradentes. Após aprovação pelo CEP em 10 de agosto de 2020, pelo parecer de número 4.203.918, a pesquisa foi realizada mantendo-se nos padrões exigidos pela instituição. Não foi necessário Termo de Consentimento Livre e Esclarecido (TCLE), pois não houve contato com o paciente, visto que se tratou-se de um estudo retrospectivo.

\section{Resultados}

No estudo foram analisados 261 prontuários dos RN egressos da UTIN, em que 105 constavam o resultado do Teste do Pezinho. Dentre as doenças triadas nota-se que a dosagem de Fenilalanina se situou dentro da normalidade em todos os pacientes; a positividade para o Hipotireoidismo Congênito foi de 3,8\%, para Fibrose Cística foi de 2\%, para Hiperplasia Adrenal Congênita foi de 1\%, para Deficiência de Biotinidase foi de 4,2\% e para as Hemoglobinopatias foi de 9,5\% (Tabela $1)$.

Tabela 1 - Resultado do Teste do Pezinho nos prontuários coletados de janeiro de 2018 a junho de 2020.

\begin{tabular}{|c|c|c|c|}
\hline $\begin{array}{l}\text { Resultado no } \\
\text { prontuário }\end{array}$ & $\mathbf{N}(\%)$ & Doenças triadas & Número absoluto \\
\hline \multirow[t]{7}{*}{ Sim } & $105(40,2 \%)$ & Hemoglobinopatias & 10 \\
\hline & & Fenilcetonúria & $\mathbf{0}$ \\
\hline & & $\begin{array}{l}\text { Hipotireoidismo } \\
\text { congênito }\end{array}$ & 4 \\
\hline & & Fibrose Cística & 2 \\
\hline & & $\begin{array}{c}\text { Hiperplasia adrenal } \\
\text { congênita }\end{array}$ & 1 \\
\hline & & $\begin{array}{l}\text { Deficiência de } \\
\text { Biotinidase }\end{array}$ & 5 \\
\hline & & Sem alterações & 83 \\
\hline Não & $183(59,8 \%)$ & & \\
\hline Total & $261(100 \%)$ & & \\
\hline
\end{tabular}

Fonte: Autores.

Ao analisar separadamente cada variável notou-se que a dosagem de Fenilalanina -utilizada para o rastreio da Fenilcetonúria- teve uma média de $0,8 \mathrm{mg} / \mathrm{dl}( \pm 0,3)$ a qual possui Valor de Referência (VR) $<2,6 \mathrm{mg} / \mathrm{dl}$. Ao dosar o Hormônio Estimulador da Tireoide (TSH) - utilizado para o rastreio do Hipotireoidismo Congênito - houve uma média de 2,4 uU/ml $( \pm 4,2)$, com valor máximo de 40uU/ml (VR: $<5,2 \mathrm{uU} / \mathrm{ml}$ ) o que permitiu identificar a existência de anormalidade em quatro pacientes. O mesmo comportamento pode ser observado para dosagem da Tripsina Imuno Reativa (IRT) - utilizada para detectar a Fibrose Cística- que apresentou valor máximo de $82 \mathrm{mg} / \mathrm{d}$ (VR: <70mg/dl), com dois pacientes apresentando alterações. A dosagem de Biotinidase -utilizada no rastreio da Deficiência de Biotinidase- teve valor máximo de 471,5 Ug/ml 
(VR: $<70 \mathrm{Ug} / \mathrm{ml}$ ) em que cinco pacientes apresentaram valor alterado. A dosagem da 17OHProgesterona - para rastreio de Hiperplasia Adrenal Congênita apresentou média de 8,3 ng/ml $( \pm 26,6)$, com apenas um paciente com alteração (Tabela 2).

Tabela 2 - Análise das variáveis das doenças triadas de janeiro de 2018 a junho de 2020.

\begin{tabular}{|c|c|c|c|c|c|c|}
\hline Variável & $\begin{array}{l}\text { Valores de } \\
\text { referência }\end{array}$ & Média & Mediana & $\begin{array}{l}\text { Desvio } \\
\text { padrão }\end{array}$ & Mínimo & Máximo \\
\hline Fenilalanina & $<2,6 \mathrm{mg} / \mathrm{dl}$ & 0,8 & 0,8 & 0,3 & 0,3 & 1,9 \\
\hline TSH & $<5,2 \mathrm{uU} / \mathrm{ml}$ & 2,4 & 1,4 & 4,2 & 0,1 & 40,0 \\
\hline IRT & $<70 \mathrm{mg} / \mathrm{dl}$ & 25,5 & 22,2 & 15,0 & 4,9 & 82,0 \\
\hline Biotinidase & $<70 \mathrm{Ug} / \mathrm{ml}$ & 183,1 & 167,2 & 85,6 & 7,8 & 471,5 \\
\hline 17 OH Prog & $\begin{array}{c}\text { Varia de } \\
\text { acordo com } \\
\text { o percentil } \\
99\end{array}$ & 8,3 & 4,2 & 26,6 & 0,4 & 262,3 \\
\hline
\end{tabular}

Fonte: Autores.

Em relação ao rastreio das Hemoglobinopatias o método utilizado foi a Eletroforese por Focalização Isoelétrica (FIE) através da análise das hemoglobinas. O padrão FA é indicativo de normalidade e esperado em recém-nascidos. Em contrapartida, todos os outros padrões existentes podem indicar alteração de hemoglobina (Tabela 3).

Tabela 3 - Rastreio das Hemoglobinopatias pelo FIE nos prontuários colhidos de janeiro de 2018 a junho de 2020.

\begin{tabular}{l|l}
\hline Padrões das hemoglobinas & $\mathbf{N} \%$ \\
\hline FA (Normal) & $\mathbf{9 0 , 5 \%}(95$ pacientes $)$ \\
\hline FA BARTS & $\mathbf{5 , 7 \%}(6$ pacientes $)$ \\
\hline FAZ & $\mathbf{3 , 8}(4$ pacientes $)$ \\
\hline
\end{tabular}

Fonte: Autores.

\section{Discussão}

O Programa de Triagem Neonatal, coloca o teste do pezinho em caráter obrigatório desde 2001, estabelecendo uma relação entre tecnologia, conhecimento e informação, culminando em diagnóstico padrão ouro precoce. Tal fato permite o controle e a redução de riscos em relação à saúde, além de levar a redução da morbidade e mortalidade e melhorar a qualidade de vida dos pacientes diagnosticados. Associa-se a este fato o benefício da redução de gastos futuros para a sociedade e para os Sistemas de Saúde (Brasil, 2016).

Grande parte das doenças identificadas na triagem neonatal se enquadra no grupo dos Erros Inatos do Metabolismo. Todas elas se caracterizam por defeitos enzimáticos e são determinadas geneticamente, com maior frequência por herança autossômica recessiva, ligada ao X ou por herança mitocondrial. Atualmente no PNTN, é possível fazer a triagem de fenilcetonúria, hipotireoidismo congênito, fibrose cística, hemoglobinopatias, hiperplasia adrenal congênita e deficiência de biotinidase, como mencionado anteriormente. As doenças triadas são passíveis de tratamento com sucesso, mas algumas delas, 
quando não diagnosticadas e tratadas precocemente, podem provocar situações graves, até mesmo causar o óbito (Rodrigues, 2019).

Houve notória evolução em relação à qualidade da coleta e da cobertura nos últimos anos. Brasil (2018) afirma que o percentual de coleta do teste na idade ideal foi de 44,85\% em 2004 para 53,51\% em 2017, sendo o maior índice de coleta em idade ideal no ano de 2011, com 61,97\% dos recém-nascidos triados até o quinto dia de vida. Já o percentual de cobertura nacional do PNTN aumentou de 74,98\% em 2004 para 85,80\% em 2017, sendo o maior índice registrado.

A fenilcetonúria (PKU) é o mais comum dos erros congênitos do metabolismo de aminoácidos, sendo uma doença de herança genética autossômica recessiva. No Brasil, a maior dificuldade na obtenção dos dados de prevalência é que não há informações centralizadas. Estudos conduzidos no Brasil sobre a incidência de fenilcetonúria em amostras de triagem neonatal demonstraram resultados diversos nas diferentes regiões do país, como as prevalências de 1:8690 aqui em Sergipe, 1:1749 em Araraquara (SP), 1:16.334 no Recôncavo Baiano, 1:19.409 em Ribeirão Preto (SP), 1:25.313 no Rio de Janeiro, 1:28.309 no Tocantins, 1:28.862 em Santa Catarina e 1:33.068 obtidos em um estudo realizado em Mato Grosso. Essa importante diferença de número de casos observada entre os Estados pode ser devida à forma de registro e ao funcionamento dos serviços de triagem neonatal. (Carvalho, et al., 2017). Na presente amostra, chama atenção a detecção nula de PKU, indo de encontro à prevalência esperada de acordo com os dados da SBTN, que pode ter explicação nos exames ainda sem resultado ou nas interferências das variáveis étnicas. Outro fator limitante e possível causador do resultado é o tamanho da amostra.

A FC é a doença hereditária mais comum em descendentes europeus. Cerca de oitenta mil pessoas são diagnosticadas com FC no mundo, no entanto também demonstra variações geográficas (Lubamba, et al., 2012). De acordo com a Cystic Fibrosis Foundation, cerca de 70 mil pessoas no mundo vivem com Fibrose Cística. Estima-se que a prevalência da doença seja de 1/3.500 até 1/10.000 nascidos vivos, dependendo da região geográfica (Santos, et al., 2005). É menos frequente em negros e rara em asiáticos. A incidência da FC varia entre as diferentes regiões do Brasil devido à heterogeneidade da população brasileira, chegando a uma prevalência de 1:15.460 em 2001 e de 1:17.091 em 2002, segundo Sociedade Brasileira de Triagem Neonatal. Vale ressaltar que a triagem neonatal para fibrose cística identifica os recém-nascidos com risco de ter a doença, mas não confirma o diagnóstico. $\mathrm{O}$ índice de testes falso-positivos pelo algoritmo baseado na quantificação de tripsinogênio imunorreativo é alto. Entretanto, a triagem neonatal negativa não exclui o diagnóstico (Smyth, et al., 2014), o que pode modificar a interpretação dos dois pacientes positivados no estudo.

A HC, patologia endócrina congênita mais frequente, foi a terceira condição com maior número de casos diagnosticados na análise. Esse diagnóstico tem uma grande importância, pelo risco de comprometimento do crescimento e desenvolvimento neurológico, que podem acarretar um estado extremo de dependência. As crianças que têm o diagnóstico precocemente estabelecido pelos Programas de Triagem Neonatal não apresentarão qualquer sintomatologia clínica, desde que a terapia de reposição hormonal seja iniciada no tempo adequado (SBP, 2018).

Após a adoção dos programas de TN o cenário da incidência mundial de hipotireoidismo congênito começou a mudar. Anteriormente, os diagnósticos eram feitos somente após as manifestações clínicas, compreendendo a uma faixa de 1:7.000 a 1:10.000. Com o advento do rastreamento de RN a incidência demonstra uma variação de 1:1500 até 1:4000, sendo duas vezes mais comum no sexo feminino (SBP, 2018). Com o decorrer dos programas de triagem, tornou-se evidente que a incidência varia de acordo com a localização geográfica e etnia. Vários programas relataram uma maior incidência em países da Ásia, nos nativos americanos e populações hispânicas e menor na população negra americana em relação à população branca. Há, também, em quase todos os relatórios dos programas de triagem, uma preponderância do sexo feminino (Rastogi, 2010). No Brasil, segundo a Sociedade Brasileira de Triagem Neonatal, foi demonstrada uma prevalência de 1:15.460 em 2001 e de 1:17.091 em 2002, sendo este o último levantamento realizado no país (Pilar, 2018).

A HAC é uma doença com significativa morbimortalidade, subdiagnosticada no período neonatal, mais comum em 
crianças do sexo masculino, antes de sua inclusão nos testes de triagem neonatal (Speiser, et al., 2010). Estudos recentes apontam para uma frequência elevada na população brasileira, em torno de 1:10000 a 1:18000 nascidos vivos (Kopacek, et al., 2017).

As hemoglobinopatias são as alterações mais comuns diagnosticadas na Triagem Neonatal. Dentre elas, a que apresenta uma prevalência e incidência relevante é a anemia falciforme. Segundo o Programa Nacional de Triagem Neonatal do Ministério da Saúde (PNTN/MS), em 2019, foram diagnosticados 1.214 casos de doença falciforme e 61.021 com Hemoglobina S (traço falciforme). Tal fato tem uma influência na prevalência direta nas hemoglobinopatias, pois sabe - se que a anemia falciforme predomina na população de raça negra, com tendência a atingir parcela cada vez maior da população devido ao alto grau de miscigenação.

$\mathrm{Na}$ análise dos nossos dados é possível perceber que as hemoglobinopatias tiveram a maior porcentagem de casos positivos. Esse dado tem um grande valor, pois sabemos que a deteç̧ão de um RN com padrão hemoglobínico alterado desencadeia uma cascata de testes nos demais membros da família, demonstrando um dos benefícios adicionais da triagem neonatal, possibilitando a detecção de doença e a investigação e o aconselhamento de outros membros da família (Sommer, 2006).

Em relação a Deficiência de Biotinidase, sabe - se que o conteúdo epidemiológico sobre a doença no Brasil é escasso. Existem poucos estudos sobre a prevalência da DB e há uma discordância nos resultados encontrados, o que torna difícil a análise dos dados em busca de uma relação com os Estados. Essa discordância diz respeito à tendência diferente em relação ao panorama mundial da doença, indicando que o Brasil pode ter uma das mais altas incidências já relatadas de DB combinada, variando de 1:6843 a 1:62.500, enquanto estudos de abrangência mundial apontam para cerca de 1:60089 nascidos vivos.

Dentro dessa pesquisa, a Deficiência de Biotinidase foi a terceira doença com mais casos positivos e merece ter sua prevalência melhor estudada, pois o paciente pode apresentar um quadro bastante diverso com alopecia, atraso no desenvolvimento, hipotonia muscular, crises epilépticas, rash cutâneo com descamação, infecções de pele, ataxia, deficiência auditiva, letargia, problemas respiratórios, anormalidades visuais e dentre outras alterações. Contudo, se diagnosticada precocemente é possível fazer o tratamento de forma simples com a administração de biotina por via oral diariamente.

\section{Conclusão}

A doença metabólica de maior prevalência nesse estudo foi o grupo que compreende as Hemoglobinopatias triadas pelo teste do pezinho, que correspondem a 9,5\% de resultados positivos. Tal achado corrobora com o perfil de prevalência de doença metabólica no Brasil, com ênfase para a Anemia Falciforme, na triagem neonatal. Em contrapartida, a menor prevalência foi da Hiperplasia Adrenal Congênita. Entretanto, se forem diagnosticadas precocemente, existe alta probabilidade de o tratamento prevenir suas graves consequências. Portanto, é fundamental que as famílias saibam que a maior parte das doenças triadas no Teste do Pezinho são assintomáticas no período neonatal

Como limitações deste estudo, encontram-se o reduzido número de prontuários com o resultado do TN e a perda de seguimento de pacientes que o realizaram. Por fim, sugere-se para trabalhos futuros, novas pesquisas acerca das doenças do TN nos demais estados da região, para efeito comparativo, além da tentativa de abranger o maior número de pacientes.

\section{Referências}

Arantes, R. R., Rodrigues, V. d. M., Norton, R. d. C., \& Starling, A.L.P. (2016). Deficiência de biotinidase: da triagem neonatal à confirmação diagnóstica e ao tratamento. Rev Med Minas Gerais, 26 (Supl 5): S48-S51. 10.5935/2238-3182.20160057

Athanazio, R. A et al. (2017). Diretrizes brasileiras de diagnóstico e tratamento da fibrose cística. Jornal Brasileiro de Pneumologia, 43(3), 219-245. https://doi.org/10.1590/s1806-37562017000000065 
Botler, J., Camacho, L. A. B., Cruz, M. M. d, \& George, P. (2010). Triagem neonatal: o desafio de uma cobertura universal e efetiva. Ciência \& Saúde Coletiva, 15(2), 493-508. https://doi.org/10.1590/S1413-81232010000200026

Brasil, Ministério da Saúde. (2011). Portaria n. 2488, de 21 de outubro de 2011.

Brasil, Ministério da Saúde. (2019). Protocolo clínico e diretrizes terapêuticas Fenilcetonúria. Relatório de recomendação, $16,6-16$.

Brasil, Ministério da Saúde (2013). Secretaria de Atenção à Saúde. Departamento de Atenção Básica. Rastreamento / Ministério da Saúde, Secretaria de Atenção à Saúde, Departamento de Atenção Básica.

Brasil, Ministério da Saúde. (2016). Secretaria de Atenção à Saúde. Departamento de Atenção Especializada e Temática, Triagem neonatal biológica: manual técnico. Brasília, DF. Ministério da Saúde.

Camargo, C. C., Fernandes, G. M. d. A., Chiepe, K. C. M. B. (2019). Doenças identificadas na triagem neonatal ampliada. Brazilian Journal of Health Review, 2, 6088-6098. https://doi.org/10.34119/bjhrv2n6-103

Carvalho, D. C. e S. N., Macedo, T. C. C., Moreno, M., Figueiredo, F. W. dos S., Sales, I. B., Christofolini, J., Bianco, B., Barbosa, C. P., \& Christofolini, D. M. (2017). Evolution of Neonatal Screening Program in a reference hospital in Ceará: 11 years of observation. ABCS Health Sciences, 42(3). https://doi.org/10.7322/abcshs.v42i3.933

Corrêa, A. L. D., Coelho, H. J., Damasceno, J. M., Farage, M. B., Zanella, D. P., Soares, J. M., Valadão, A. F. V. (2019). Prevalência das doenças triadas no teste do pezinho no município de Timóteo-MG. Brazilian Journal of Surgery and Clinical Research - BJSCR, 25(2), 48-52.

Cruz, R.D.C.M. (2014). A importância do teste do pezinho para o conhecimento das mães. Saberes Unicampo, 1(1), 67-69.

de Marqui, A. B. T. (2017). Fenilcetonúria: aspectos genéticos, diagnóstico e tratamento. Revista da Sociedade Brasileira de Clínica Médica, 15(4), 282-288.

Engelgau M, Narayan K, Herman W. (2000). Screening for type 2 diabetes. Diabetes Care, United States, 23(10), 1563-1580.

Guttler, F., \& Guldberg, P. (1996). The influence of mutations of enzyme activity and phenylalanine tolerance in phenylalanine hydroxylase deficiency. European journal of pediatrics, 155(1), S6-S10. https://doi.org/10.1007/p100014253.

Kopacek, C., de Castro, S. M., Prado, M. J., da Silva, C. M., Beltrão, L. A., \& Spritzer, P. M. (2017). Neonatal screening for congenital adrenal hyperplasia in Southern Brazil: a population based study with 108,409 infants. BMC pediatrics, 17(1), 22. https://doi.org/10.1186/s12887-016-0772-x

Kohne E. (2011). Hemoglobinopathies: clinical manifestations, diagnosis, and treatment. Deutsches Arzteblatt international, 108(31-32), 532-540. https://doi.org/10.3238/arztebl.2011.0532

LaFranchi, S. H., \& Austin, J. (2007). How should we be treating children with congenital hypothyroidism. Journal of pediatric endocrinology \& metabolism: JPEM, 20(5), 559-578. https://doi.org/10.1515/jpem.2007.20.5.559

Leão, L. L., \& Aguiar, M. J. B. d. (2008). Triagem neonatal: o que os pediatras deveriam saber. Jornal de Pediatria, 84(4, Suppl.), S80-S90. https://doi.org/10.1590/S0021-75572008000500012

Levy H. L. (1998). Newborn screening by tandem mass spectrometry: a new era. Clinical chemistry, 44(12), 2401-2402.

Lubamba, B., Dhooghe, B., Noel, S., \& Leal, T. (2012). Cystic fibrosis: insight into CFTR pathophysiology and pharmacotherapy. Clinical biochemistry, 45(15), 1132-1144. https://doi.org/10.1016/j.clinbiochem.2012.05.034

Maciel, L. M. Z., Kimura, E. T., Nogueira, C. R., Mazeto, G. M. F. S., Magalhães, P. K. R., Nascimento, M. L., Nesi-França, S., \& Vieira, S. E. (2013).

Hipotireoidismo congênito: recomendações do Departamento de Tireoide da Sociedade Brasileira de Endocrinologia e Metabologia. Arquivos Brasileiros de Endocrinologia \& Metabologia, 57(3), 184-192. https://doi.org/10.1590/S0004-27302013000300004

Pereira A.S. et al. (2018). Metodologia da pesquisa científica. UFSM.

Pilar, B. C. (2018). Triagem neonatal: aspectos clínicos e laboratoriais. Brazilian Journal of Clinical Analyses, $50(2), 30$.

Rastogi, M. V., \& LaFranchi, S. H. (2010). Congenital hypothyroidism. Orphanet journal of rare diseases, 5, 17. https://doi.org/10.1186/1750-1172-5-17

Rodrigues, L. P., Tanaka, S. C. S. V., Haas, V. J., Cunali, V. C. A., \& Marqui, A. B. T. d. (2019). Teste do pezinho: condições materno-fetais que podem interferir no exame em recém-nascidos atendidos na unidade de terapia intensiva. Revista Brasileira de Terapia Intensiva, 31(2), 186-192. https://doi.org/10.5935/0103-507x.20190030

Santos, G. P. C., Domingos, M. T., Wittig, E. O., Riedi, C. A., \& Rosário, N. A. (2005). Programa de triagem neonatal para fibrose cística no estado do Paraná: avaliação após 30 meses de sua implantação. Jornal de Pediatria, 81(3), 240-244. https://doi.org/10.2223/JPED.1333

SBP, Sociedade Brasileira de Pediatria (2018). Hipotireoidismo Congênito: Triagem Neonatal. Departamento Científico de Endocrinologia.

SBP, Sociedade Brasileira de Pediatria (2019). Hiperplasia adrenal congênita: triagem neonatal. Departamento Científico de Endocrinologia.

Silva, C. d. A., Baldim, L. B., Nhoncanse, G. C., Estevão, I. d. F., \& Melo, D. G. (2015). Triagem neonatal de hemoglobinopatias no município de São Carlos, São Paulo, Brasil: análise de uma série de casos. Revista Paulista de Pediatria, 33(1), 19-27. https://doi.org/10.1016/j.rpped.2014.08.001

Silva, M. P. C., Contim, D., Ferreira, L. A., \& Marqui, A. B. T. d. (2017). Teste do pezinho: percepção das gestantes nas orientações no pré-natal. Revista Brasileira de Saúde Materno Infantil, 17(2), 291-298. https://doi.org/10.1590/1806-93042017000200005 
Research, Society and Development, v. 10, n. 5, e45010514907, 2021

(CC BY 4.0) | ISSN 2525-3409 | DOI: http://dx.doi.org/10.33448/rsd-v10i5.14907

Smith, C. et al (2007). Bioquímica Médica Básica de Marks: Uma Abordagem Clínica. (2a ed.), Artmed.

Sommer, C. K., Goldbeck, A. S., Wagner, S. C., \& Castro, S. M. (2006). Triagem neonatal para hemoglobinopatias: experiência de um ano na rede de saúde pública do Rio Grande do Sul, Brasil. Cadernos de Saúde Pública, 22(8), 1709-1714. https://dx.doi.org/10.1590/S0102-311X2006000800019

Smyth, A. R. et al (2014). European Cystic Fibrosis Society Standards of Care: Best Practice guidelines. Journal of cystic fibrosis: official journal of the European Cystic Fibrosis Society, 13, S23-S42. https://doi.org/10.1016/j.jcf.2014.03.010

Speiser, P. W. et al (2010). Congenital adrenal hyperplasia due to steroid 21-hydroxylase deficiency: an Endocrine Society clinical practice guideline. The Journal of clinical endocrinology and metabolism, 95(9), 4133-4160. https://doi.org/10.1210/jc.2009-2631

Tesser C. D. (2017). Why is quaternary prevention important in prevention? Revista de saude publica, 51, 116. https://doi.org/10.11606/S15188787.2017051000041

Wollf B. (2001). Disorders of biotin metabolism. In: Scriver CR, Beaudet AL, Sly Ws, Valle D. The Metabolic and Molecular Bases of Inherited Diseases. (8a ed.), McGraw-Hill, p. 3935-62.

Zhu, Meilin. (2017). "The Guthrie Test for Early Diagnosis of Phenylketonuria". Embryo Project Encyclopedia. http://embryo.asu.edu/handle/10776/11460.

Zilka, L. J., Lott, J. A., Baker, L. C., \& Linard, S. M. (2008). Finding blunders in thyroid testing: experience in newborns. Journal of clinical laboratory analysis, 22(4), 254-256. https://doi.org/10.1002/jcla.20247 